\title{
In Situ Electrochemical Deposition of Poly(3,4-ethylenedioxythiophene) (PEDOT)
}

\author{
Jinglin Liu ${ }^{1}$, Bin Wei ${ }^{1}$, Jennifer Sloppy ${ }^{1}$, Liangqi Ouyang ${ }^{1}$, Chaoying $\mathrm{Ni}^{1}$ and David C. Martin ${ }^{1,2}$ \\ 1. Department of Materials Science and Engineering, The University of Delaware, Newark, DE 19716, \\ USA. \\ 2. Department of Biomedical Engineering, The University of Delaware, Newark, DE 19716, USA.
}

Conjugated polymers are widely used in organic solar cells, chemical sensors and biomedical devices because of their relatively high conductivity and soft mechanical properties [1,2,3]. Electrochemical deposition has long been an important method of fabricating conjugated polymer thin films for various applications. The morphology of the films, and the corresponding performance of the devices is particularly sensitive to the detailed fabrication conditions. Previous efforts have investigated the nucleation and growth mechanisms of electropolymerized conjugated polymers, however the results are usually obtained from in-direct and lower resolution methods like UV-vis and AFM [4,5]. More detailed information with ultra-high resolution is still needed. Electrochemical liquid electron microscopy makes it possible to analyze this initial nucleation and growth process in direct imaging method in a TEM with nanometer scale resolution. This method has proven effective for the analysis of electrochemical deposition mechanisms in copper and lead [6,7]. No previous studies have examined the in situ TEM electrochemical deposition of conjugated polymers, mostly probably due to the fact that polymers are highly electron beam sensitive. This high sensitivity makes the in situ TEM imaging of conjugated polymer electrodeposition much more experimentally difficult than for inorganic materials.

Of all the conjugated polymers, poly(3,4-ethylenedioxythiophene) (PEDOT) has been extensively studied for its high conductivity and stability, and has been utilized in various applications. PEDOT was chosen here as a model system to demonstrate the feasibility of performing electrochemical deposition of conjugated polymers within the TEM [8].

The in situ imaging of electrochemical deposition of PEDOT was conducted on a $300 \mathrm{kV}$ JEOL 3010 electron microscope with a Protochips Poseidon 500 electrochemical liquid flow holder. Ethylenedioxythiophene (EDOT) monomer solution with counter-ion electrolyte $\left(\mathrm{LiClO}_{4}\right)$ was used in continuous flow mode, where the liquid layer was sealed between two microfabricated silicon chips. Thin silicon nitride windows $(\sim 50 \mathrm{~nm})$ on the chips allow the electron beam to pass through for TEM imaging. The liquid layer thickness was defined by the spacers on bottom chips. Electrochemical deposition and measurements were carried out with a Gamry Reference 600 potentiostat/galvanostat. A $20 \mu \mathrm{m}$ wide glassy carbon working electrode was in the middle of the membrane with a platinum reference electrode around working electrode and remotely counter electrode (Figure 1a).

Effective electrical contact was tested using cyclic voltammetry (CV) and successful PEDOT deposition from both $\mathrm{CV}$ and constant voltage (potentiostatic) methods were confirmed from TEM imaging. PEDOT was found to nucleate first near the edges of the working electrode (Figure 1b). With further deposition, the initial clusters increased in both size and thickness, along with additional nucleation sites seen in the middle of the working electrode. Liquid-like fluctuating domains were observed during deposition and of a size and rounded shape consistent with the bumpy surface structure seen in SEM images of electrochemically polymerized PEDOT thin films. After the experiment, the electrochemical chip was taken out for correlative optical microscopy (OM) and scanning electron microscopy (SEM) 
imaging. The dark color under OM, the bumpy surface structure seen in the SEM, and the strong sulphur signal from EDS elemental mapping all confirmed the successful deposition of PEDOT (Figure 1c).

In conclusion, the in situ electrochemical deposition of conjugated polymers was successfully conducted and imaged under TEM with an electrochemical liquid flow cell for the first time. This new technique provides an unparalleled method for directly investigating the early stage nucleation and growth mechanisms of electropolymerized conjugated polymer thin films. This information will be of great value in understanding the performance of conjugated polymer thin films and in optimizing processing conditions for devices with enhanced properties [9].

References:

[1] S. Günes, H. Neugebauer and N. S. Sariciftci, Chemical reviews, volume 107, no. 4 (2007), p. 13241338.

[2] K. Lee, J. M. Rouillard, T. Pham, E. Gulari and J. Kim, Angewandte Chemie, volume 46, no. 25 (2007), p. 4667-4670.

[3] X. Cui, V. A. Lee, Y. Raphael, J. A. Wiler, J. F. Hetke, D. J. Anderson and D. C. Martin, Journal of biomedical materials research, volume 56, no. 2 (2001), p. 261-272.

[4] A. R. Hillman, and E. F. Mallen, Journal of Electroanalytical Chemistry, volume 243 (1988), p. 403-417.

[5] M. Innocenti, F. Loglio, L. Pigani, R. Seeber, F. Terzi and R. Udisti, Electrochimica Acta, volume 50 (2005), p. 1497-1503.

[6] A. Radisic, P. M. Vereecken, J. B. Hannon, P. C. Searson and F. M. Ross, Nano Letters, volume 6, no. 2 (2006), p. 238-242.

[7] E. R. White, S. B. Singer, V. Augustyn, W. A. Hubbard, M. Mecklenburg, B. Dunn and B. C. Regan, ACS Nano, volume 6, no. 7 (2012), p. 6308-6317.

[8] J. Liu, B. Wei, J. Sloppy, L. Ouyang, C. Ni and D. C. Martin, in preparation.

[9] The authors acknowledge funding from the National Science Foundation, Grant Number 1103027 and the University of Delaware.
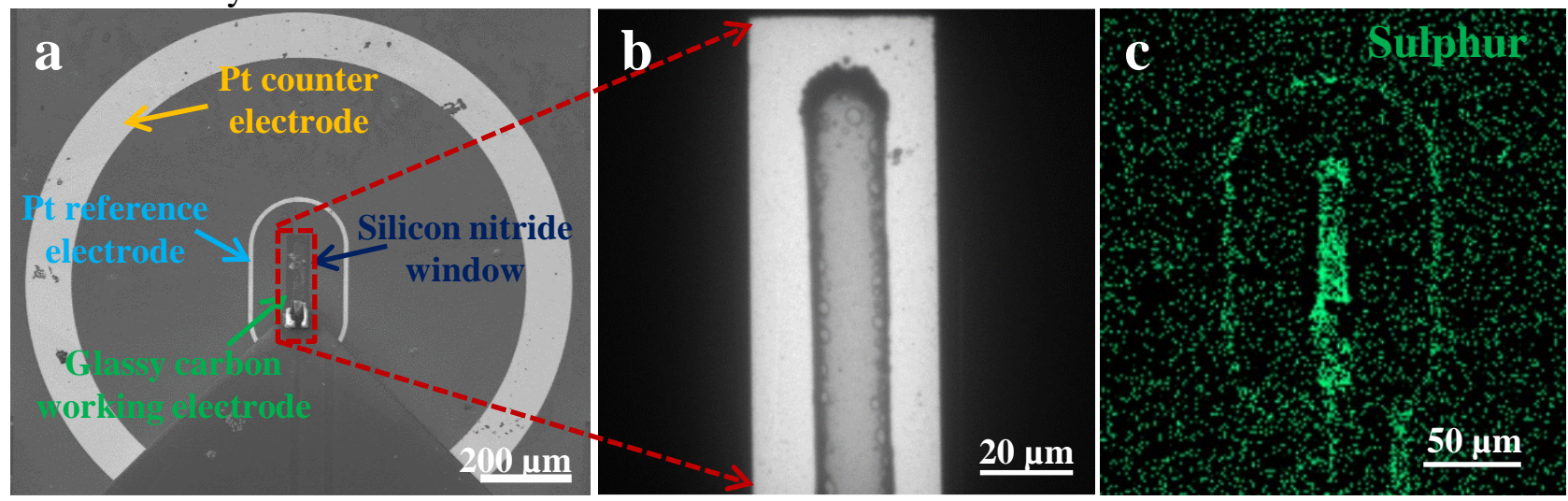

Figure 1. a: Scanning electron micrograph of the electrochemical top chip showing the middle silicon nitride window for imaging with glassy carbon working electrode, Pt reference and counter electrode for electrochemical deposition and measurements. b: Transmission electron micrograph showing early stage PEDOT deposition around the edges of glassy carbon working electrode. c: Elemental map for sulphur signal confirming the successful deposition of PEDOT. 